\title{
Transmission Grid Expansion Planning Method Research and Implementation
}

\author{
Wenjie Zheng1,a, Jiajian Huang1 \\ 1Electric Power Research Institute of Guangdong Power Grid \\ Corporation, Guangdong Provincial Key Laboratory of Smart Grid \\ technology, Guangzhou, 510080, China \\ aemail: richard_zwj@sina.com
}

\begin{abstract}
The transmission expansion planning (TEP) problem aiming to improve the reliability of the power system is of great importance. In this paper, a DC power flow model with reliability is proposed for TEP. In the proposed model, three reliability indices are embedded into the objective. The reliability of transmission grid is considered for the first time to guide the expansion planning. To solve the model, a novel multi swarm based fruit fly optimization algorithm (FOA) is proposed. In addition, the new model and the proposed algorithm are applied to IEEE reliability test system RTS-79. Finally, numerical results demonstrate the validity of new model and the effectiveness of the proposed FOA.
\end{abstract}

Keywords: Transmission Expansion Planning; Reliability; DC Power Flow Model; Fruit Fly Optimization Algorithm

\section{Introduction}

The fast development of national economics is impossible without the support of the power systems. The operating reliability of the power system concerns whether the social and economic activities can perform normally. Generally, the transmission grid structure has a direct impact on the reliability of the power system. Although the power transmission grid structure is well planned, it happens inevitably that new transmission lines should be built to meet the increasing demand capacity of the power with the expanding of the economic activity and relieve congestion in the existing network. Therefore, the power transmission expansion planning based on the original transmission grid structure to enhance the overall system reliability and market efficiency is of great significance.

In the last decades, the power transmission expansion planning (TEP) problem has attracted tremendous attention in the field of power system. As for the modeling for TEP, various TEP models have been proposed [1], mainly including DC network model [2-4] and AC network model [5]. Compared with the extensively studied DC model, there are only a few works about AC model due to the complexity of nonlinear and non-convex. As for the solution methods 
for TEP problem, two main types of approaches were proposed, i.e. mathematic programming methods and heuristic methods. The former type of method was usually applied to solve the TEP problem with small scale, and the TEP was modeled as a linear programming problem, such as [2], [3]. Recently, heuristic methods have become prevailing since heuristics can find good feasible solutions even if they are applied to solve the large scale, non-linear and non-convex TEP problem. These heuristics included genetic algorithm [6], particle swarm optimization [6], shuffled frog leaping algorithm [6].

Though the researches about TEP problem were abundant, the optimization objectives were almost all focused on the operation cost or investment. The reliability of transmission grid was seldom considered in the objectives. The common consideration about the reliability was the $\mathrm{N}-1$ reliability constraint [4]. As the development of power system, the reliability is getting more and more important to guarantee the safe operation. Now that traditional TEP ignored the reliability in designing the expanded transmission lines, three reliability indices are embedded into the optimization objective and a new DC network model is proposed for TEP problem in this work. To solve the model, a novel multi swarm based fruit fly optimization algorithm (FOA) is presented.

\section{Proposed Model}

In this section, three reliability indices of power system are introduced and then the reliability indices are embedded into the optimization objective of TEP.

\section{A. Reliability indices}

Generally, three basic reliability indices are used to evaluate the reliability of power system [7], that is, loss of load probability (LOLP), loss of load expectation (LOLE), and expected demand not supplied (EDNS).

LOLP indicates the possibility of loss-of-load resulted from under-capacity, which is calculated as:

$$
L_{L O L P}=\sum_{x \in X} I_{f}(x) P(x)
$$

(1)

where $I_{f}(x)=1$, if $x$ is the failure state; otherwise, $I_{f}(x)=0$, $P(x)$ is probability of state $x$.

LOLF indicates average times of blackout each year, which is calculated as:

$$
L_{L O L F}=\sum_{x \in X}\left\{I_{f}(x) \sum_{k=1}^{m} \lambda_{x}^{i n}(k)\right\} P(x)
$$

where $\lambda_{x}^{i n}(k)=\mu_{k}$ if the component $k$ is in the failure state; otherwise $\lambda_{x}^{i n}(k)=-\lambda_{k} . \quad \mu_{k}$ and $\lambda_{k}$ are the failure probability and 
failure frequency of component $k$, respectively.

EDNS indicates the power demand not supplied each year, which is calculated as:

$$
L_{E D N S}=\sum_{x \in X} I_{f}(x) L_{c}(x) P(x)
$$

(3)

where $L_{c}(x)$ is the minimum load reduced to restore the power system in the failure state of $x$.

\section{B. New objective}

Both the reliability of the system and the expansion cost are considered simultaneously in the new objective.

Max : $\left(w_{1} \times L_{L O L P}+w_{2} \times L_{L O L F}+w_{3} \times L_{E D N S}\right) /\left(w_{4} \times f_{c}\right)$

(4)

where $f_{c}$ is the line investment cost of expansion, which can be calculated as $f_{c}=T L_{n l} \times C_{u}, T L_{n l}$ is the total length of new built lines and $T L_{n l}=\sum_{i=1}^{n} \sum_{j=i}^{n} Z_{i j} L_{i j}, C_{u}$ is the unit price of building lines, and $w_{1} \sim w_{4}$ are four coefficients.

\section{Proposed FOA}

\section{A. Overview of FOA}

The FOA [8] is a newly proposed swarm based interactive evolutionary method. Since proposed, it has received much attention due to its easy to understand and implement and it has been successfully applied to different fields [9], such as financial distress, web auction logistics service, semiconductor final testing problem, steelmaking casting problem. The FOA consists of two main search procedures: osphresis foraging phase and vision foraging phase. The detailed procedure of the FOA can be shown as follows.

Step 1. Set the maximum number of generations and population size.

Step 2. Randomly initialize the swarm location within the search space.

Step 3. NP food sources around the swarm location are randomly generated to form a population.

Step 4. Calculate the smell concentration values of NP food sources and evaluate the population.

Step 5. Find out the best food source with the maximum smell concentration value, and then the fruit fly swarm will fly towards the location of the best food source.

Step 6. FOA ends if the stopping criterion condition is met; otherwise, go 
back to Step 3.

\section{B. Proposed FOA for TEP}

The original FOA is proposed for a two-dimension continuous optimization problem, which cannot be used directly for the TEP problem. In this subsection, a novel multi-swarm based discrete fruit fly optimization algorithm is proposed. The novel FOA consists of 4 main procedures: fruit fly swarm location initialization, ophresis foraging phase, evaluation and vision foraging phase.

Representation: Each solution in the FOA is represented by a binary value matrix $Z=\left[Z_{i j}\right]_{n} \times_{n}(i, j=1,2, \ldots, n)$, where $n$ is the number of buses in the transmission grid. The swarm location and food sources in the FOA represent solutions.

Initialization: The original FOA has the potential of exploiting the promising solution space, while the exploration is not sufficient, especially for the complicated problem. To enhance the exploration of FOA, a multi-swarm strategy is adopted. To be specifically, $M$ fruit fly swarms are used and the swarm location $Z^{k}(k=1,2, \ldots, M)$ is initialized as follows: generate a random number $r p \in(0,1)$, if $r p>0.5, Z_{i j}^{k}=1(i, j=1,2, \ldots, n)$; otherwise, $Z_{i j}^{k}=0$.

Ophresis foraging phase: NP food sources are generated around each swarm location to form the subpopulation. The food source $Z^{k, s}(k=1,2, . ., M, s=1,2, \ldots, N P)$ is generated as: randomly choose one line $i_{1}-j_{1}$, and $Z_{i_{1} j_{1}}^{k, s}=1-Z_{i_{1} j_{1}}^{k}$; for other lines $i-j\left(i \neq i_{1}, j \neq j_{1}\right), \quad Z_{i j}^{k, s}=Z_{i j}^{k}$.

Evaluation: The smell concentration value of each solution $S_{c}\left(Z^{k, s}\right)=$ fitness $\left(Z^{k, s}\right)$, where fitness() is the objective function in (5). Since the generated solutions in the ophresis foraging phase are not feasible guaranteed, the evaluation of solution should take the constraint into consideration. In the FOA, a constraint violation based priority (CVP) rule is adopted for the evaluation. For any two solutions:

1. The feasible solution is better than the infeasible one.

2. If both of two solutions are feasible or infeasible, the solution with larger fitness value.

Vision foraging phase: for each subpopulation, find out the best food source $Z^{k, \text { best }}(k=1,2, \ldots, M)$ using the constraint violation based priority rule, and the swarm location is replaced with the found best food source $Z^{k}=Z^{k, \text { best }}(k=1,2, \ldots, M)$, if $Z^{k, \text { best }}$ is better than $Z^{k}$; otherwise , keep unchanged.

\section{Simulation results}

The work presented in this work is coded with Matlab language, and the simulations are all done on a PC with an i5 CPU at 2.3GHz. To confirm the validity of the new model and the effectiveness of the proposed algorithm, the method is applied to the IEEE reliability test system RTS-79 [10], as is shown in Fig. 1. The system contains 24 bus nodes, including 10 nodes at voltage level 
$230 \mathrm{Kv}$ and 14 nodes at voltage level $138 \mathrm{Kv}$. The cost to build new transmission line with $138 \mathrm{Kv}$ and $230 \mathrm{Kv}$ is $769.2 \mathrm{KRMB} / \mathrm{Km}$ and $1341.7 \mathrm{KRMB} / \mathrm{Km}$ respectively.

The optimization result is obtained by applying the proposed model and FOA and the final expansion plan is to build the transmission line between Bus 11 and Bus 15 with the total construction cost $7692 \mathrm{RMB}$ with the line length of $10 \mathrm{~km}$. The TEP result of RTS 79 is illustrated in Fig. 2, where the dashed line is the expanded line. Before expansion, the reliability evaluation of the system is as follows: $\mathrm{LOLP}=3.32 \mathrm{E}-4, \mathrm{LOLF}=0.111$ and $\mathrm{EDNS}=0.01432$. After the expansion, the reliability of the system improves, and LOLP=2.0716E-4, LOLF=0.059 and ENDS $=0.012$. From the result, it can be concluded that the proposed reliability targeted expansion planning method can provide transmission expansion plan with low cost while improve the reliability of the system.

\section{Conclusion}

This paper presented a new DC power flow model for TEP problem. In the proposed model, the reliability of power system was considered and three reliability indices were embedded into the objective. Then, a novel multi swarm based fruit fly optimization algorithm was proposed to solve the new model. A binary value matrix was employed to represent a solution. During the osphresis foraging phase, randomly chose one possible new line and changed the planning of this line. During the vision foraging phase, the constraint violation based priority rule was adopted to find out the best food source. In addition, the validity of the proposed model and the effectiveness of FOA were demonstrated with numerical results on IEEE test power system RTS-79. The reliability of the system improves using the proposed expansion planning method. The future work is to develop multi-objective TEP model and propose corresponding solution method. 


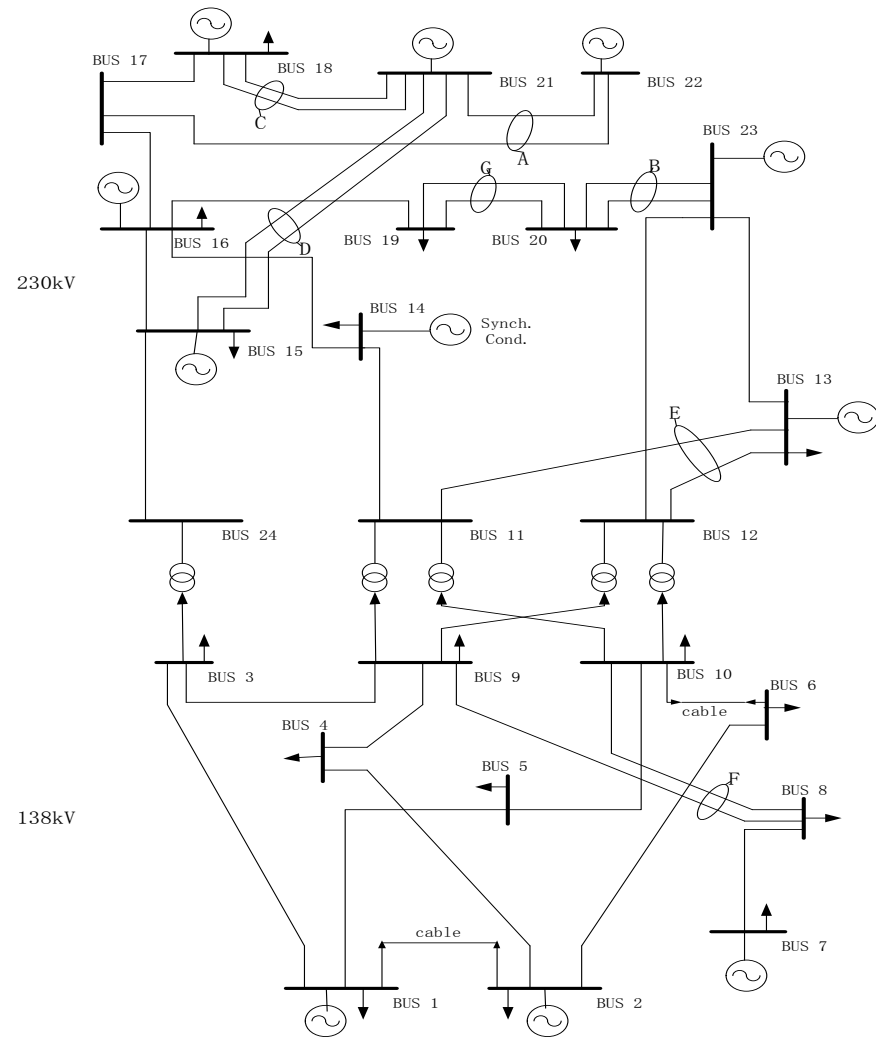

Fig.1. Transmission network of IEEE RTS-79 


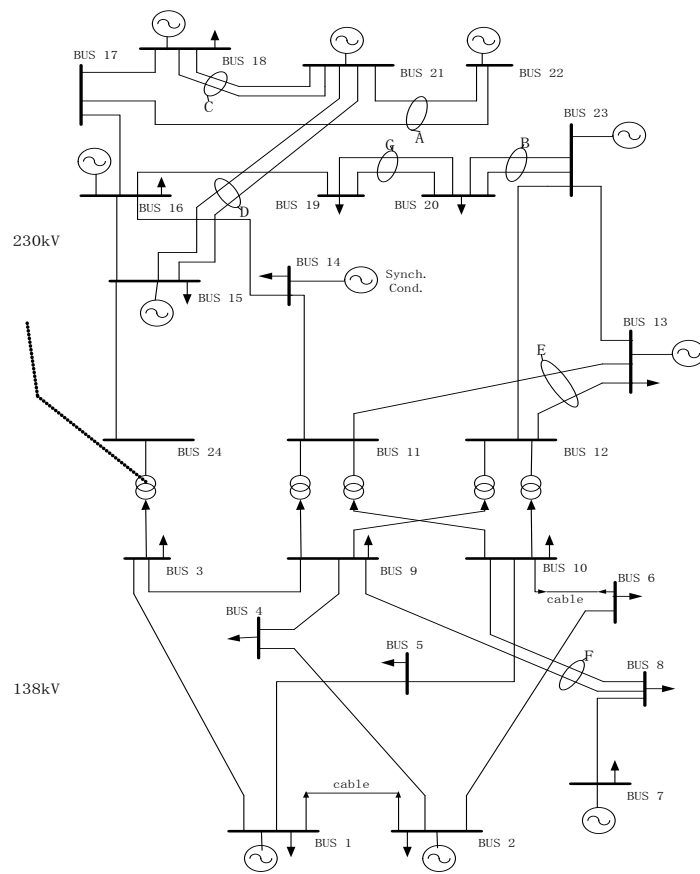

Fig.2. TEP result of IEEE RTS-79

\section{References}

[1] G. Latorre, R. D. Cruz, J. M. Areiza, A. Villegas. Classification of publications and models on transmission expansion planning [J], IEEE Trans. Power Syst., 200318 (2) 938-946.

[2] L. Bahiense, G. C. Oliveira, M. Pereira, S. Granville. A mixed integer disjunctive model for transmission network expansion [J], IEEE Trans. Power Syst., 200116 (3) 560-565.

[3] N. Alguacil, A. L. Motto, A. J. Conejo. Transmission expansion planning: a mixed-integer LP approach [J], IEEE Trans. Power Syst., 200318 (3) 1070-1077.

[4] H. Zhang, V. Vittal, G. T. Heydt, J. Quintero. A mixed-integer linear programming approach for multi-stage security-constrained transmission expansion planning [J], IEEE Trans. Power Syst., 201227 (2) 1125-1133.

[5] M. J. Rider, A. V. Garcia, R. Romero. Power system transmission network expansion planning using AC model [J], IET Gener. Transm. Distrib., 20071 (5) 731-742.

[6] E. Mehdi, T. K. Saha, K. N. Hasan. Transmission expansion planning by meta-heuristic techniques: A comparison of Shuffled Frog Leaping Algorithm, PSO and GA [C]. IEEE Power and Energy Society General Meeting. 2011. 1-8. 
[7] R. Billinton, R. N. Allan. Reliability Evaluation of Power Systems (second edition). New York and London: Plenum Press, 1996.

[8] W. T. Pan. A new fruit fly optimization algorithm: taking the financial distress model as an example [J]. Knowledge-Based Systems, 201226 69-74.

[9] X. L. Zheng, L. Wang, S. Y. Wang. A novel fruit fly optimization algorithm for the semiconductor final testing scheduling problem [J]. Knowledge-Based Systems, 201457 95-103.

[10] C. Grigg, P. Wong, P. Albrecht, et al. The IEEE reliability test system-1996. A report prepared by the reliability test system task force of the application of probability methods subcommittee [J]. IEEE Trans. Power Syst., 199914 (3) $1010-1020$. 